\title{
Thermal analysis kinetics of Tartary buckwheat flour
}

\author{
Haiyan Huang*, Jilin Li, Hong Liu \\ Xichang University, Xichang 61500, China \\ Corresponding Author Email: 34362380@qq.com
}

https://doi.org/10.18280/ijht.360433

Received: 19 February 2018

Accepted: 25 May 2018

\section{Keywords:}

tartary buckwheat flour, differential thermal analysis (DTA), thermal analysis kinetics

\begin{abstract}
This experiment uses a DTA-TG analyzer to perform thermal analysis on Tartary buckwheat flour under static-air condition. The best experimental conditions for the thermal analysis of Tartary buckwheat flour are: sample mass $3.000 \mathrm{~g}$, heating rate $10^{\circ} \mathrm{C} / \mathrm{min}$. This paper studies the thermostabilization of Tartary buckwheat flour and concludes the four stages of thermal decomposition of Tartary buckwheat flour via extrapolated onset temperature of the thermogravimetric curve, as well as the proper processing temperature for Tartary buckwheat flour should be lower than $266.74{ }^{\circ} \mathrm{C}$. By comparing Stava-Sestak method and FWO method, we can get that the apparent activation energy of the thermal decomposition of Tartary buckwheat flour is $235.38 \mathrm{KJ} / \mathrm{mol}$, and the frequency factor is $\mathrm{LnA}=44.07$. Through comparison between 30 mechanistic function models and kinetic mode function models, it infers the most probable mechanism function for simulating the thermal decomposition of Tartary buckwheat flour. The reaction kinetics model of the decomposition of Tartary buckwheat flour is preliminarily calculated, which has provided a theoretical basis for the temperature control of Tartary buckwheat flour during the processing.
\end{abstract}

\section{INTRODUCTION}

Tartary buckwheat is a kind of coarse grain which has been proved by many literatures to have health care function [1]. It is widely cultivated in the western part of China and has great development values as people are paying more attention to the health nowadays. However, in current China, the development of Tartary buckwheat is still in its infancy compared to other developed countries, and there are few studies on the modern process parameters, resulting in the current Tartary buckwheat products have many problems in the $R \& D$ and actual production process, such as the change of the properties of active substances due to improper control of processing temperature during processing weakens the health-care functions of the Tartary buckwheat products [2]. At present, the application of Tartary buckwheat in food has involved aspects of processing methods, processing characteristics, influence mechanism and processing technology, and the influence of enzyme chemistry on the quality of processed products. However, the research and application of DTA technology in Tartary buckwheat processing is rare [3].

DTA [4] is a thermobalance technology that uses programs to control the heating rate and sensitivity, by recording the mass and energy change of a substance during heating, it determines the temperature at which the substance begins to lose weight and obtains this substance's thermal stability. Through non-isothermal kinetics, it can calculate the substance's activation energy and frequency factor so as to determine the mechanism function of its thermal decomposition reaction and predict its thermal decomposition reaction model, which provides a reliable theoretical reference for the Tartary buckwheat flour in the processing and storage process. The apparent activation energy, frequency factor and mechanism function obtained are also important theoretical parameters in the in-depth study or processing of Tartary buckwheat flour.

\section{MATERIALS AND METHODS}

\subsection{Test materials and equipment}

Tartary buckwheat flour was purchased from Hangfei Tartary buckwheat Co., Ltd., moisture content is less than $10 \%$. SHMADZU DTG-60 DTA-TG analyzer: Shimadzu Corporation, Japan. The heating range is $20^{\circ} \mathrm{C} \sim 600^{\circ} \mathrm{C}$; the atmosphere is static air; the reference material is an empty ceramic crucible.

\subsection{Test methods}

2.2.1 Method for determining the best thermal analysis image

By comparing different sample masses, the corresponding thermal analysis images are first obtained, then the sharpness and apparent degree of the peak shape are also compared to obtain the best thermal analysis image, and this mass is taken as the best sample mass, again by comparing different heating rates we can obtain different thermal analysis images, and the same standard is used to obtain the best thermal analysis conditions and thermal analysis images.

\subsubsection{Determination of reaction critical temperature}

The onset temperature of the Tartary buckwheat flour is determined by the best thermal analysis image obtained and the extrapolated onset temperature of the thermogravimetric curve. 


\subsubsection{Solving thermal analysis kinetic factors}

(1) Determination of activation energy $\mathrm{E}$ and frequency factor $\mathrm{A}$

The FWO method is used to substitute the temperature of the equal conversion rate under different heating rate conditions in to the FWO equation to solve the activation energy and the frequency factor [5].

(2) Determination of the mechanism function $\mathrm{G}(\alpha)$

Each mechanism function is substituted into the SatavaSestak equation to solve the activation energies Es and As, and compare them with the activation energy $\mathrm{E}$ obtained by the FWO method. Select a kinetic mode function that satisfies the following condition: [6]

$\left|\frac{E_{0}-E_{s}}{E_{0}}\right| \leq 0.3$

Then, determine the optimal mechanism function by calculating the correlation coefficient.

\section{RESULTS AND ANALYSIS}

\subsection{Determination of the best DTA-TGA image}

3.1.1 Influence of Tartary buckwheat flour sample mass on TGA curve

In this paper, a set of five samples were prepared with a gradient of $1.000 \mathrm{~g}$ between $1.000 \mathrm{~g}$ and $5.000 \mathrm{~g}$, and the heating rate was controlled at $10{ }^{\circ} \mathrm{C} / \mathrm{min}$. By selecting the mass with sharp and obvious peak, the best sample mass suitable for the measuring of TGA curve was selected. The mass change has no significant effect on the TGA curve, but has a great influence on the DTA curve, and all curves showed three peaks, among which the peaks of the $3.000 \mathrm{~g}$ sample are more obvious and easier to analyze. By comparison, $3.000 \mathrm{~g}$ was finally selected as the best sample mass for the thermal analysis test of Tartary buckwheat flour.

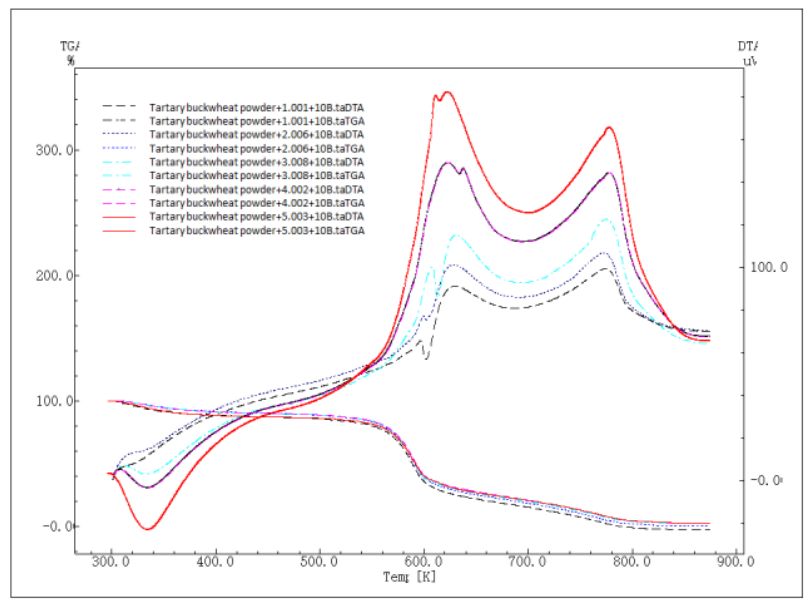

Figure 1. Influence of different sample masses on DTA/TGA curves

\subsubsection{Influence of heating rate on thermal analysis image}

The faster the heating rate, the more severe the temperature lag, and the initial and end temperature of the weight loss will lag and the information of some intermediate product might lost [7]. In this paper, by comparing the influence of heating rate on Tartary buckwheat flour, the temperature gradients of $5,10,15$, and $20^{\circ} \mathrm{C} / \mathrm{min}$ were respectively set at a mass of $3.000 \mathrm{~g}$ to obtain the most suitable DTA/TGA curve for observation and calculation.

The slower the heating rate, the earlier the energy peak of the DTA curve appears. This is related to the sensitivity of the instrument. The slower the heating rate, the steeper the curve from $300 \mathrm{~K}$ to $700 \mathrm{~K}$. It can be seen from the comparison of the DTA curve that the peak shape at a rate of $10^{\circ} \mathrm{C} / \mathrm{min}$ is clear and obvious, so the best heating rate for the differential thermal analysis of the Tartary buckwheat flour is $10{ }^{\circ} \mathrm{C} / \mathrm{min}$. In summary, the best conditions for the final selection of the Tartary buckwheat flour heat analysis test are: the heating rate is $10^{\circ} \mathrm{C} / \mathrm{min}$, and the sample mass is $3.000 \mathrm{~g}$.

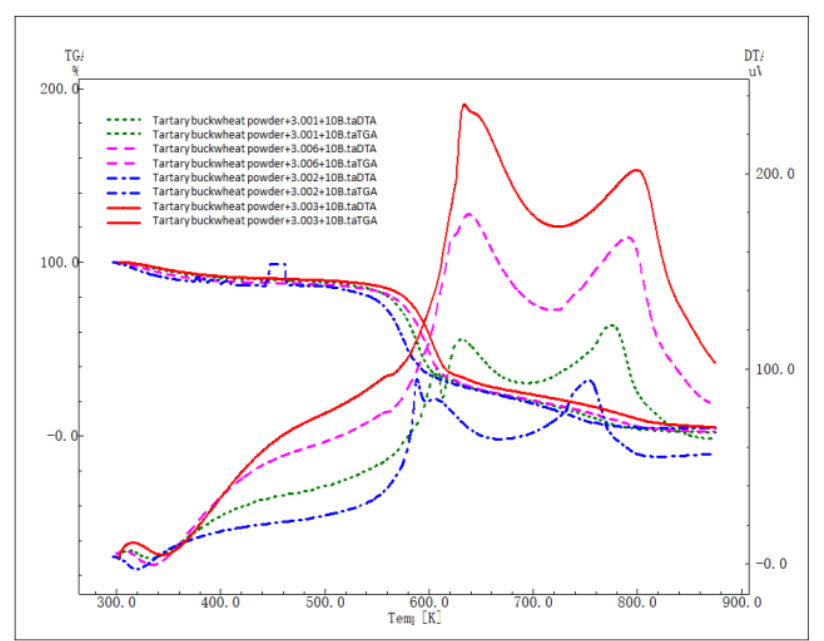

Figure 2. Influence of different heating rates on DTA/TGA curves

\subsection{Analysis of the thermal decomposition process of Tartary buckwheat flour}

The DTA and TGA curves were drawn by the preliminary experiment, and the extrapolated onset temperature of each reaction was found using TA60 software [8] as shown in the figure below.

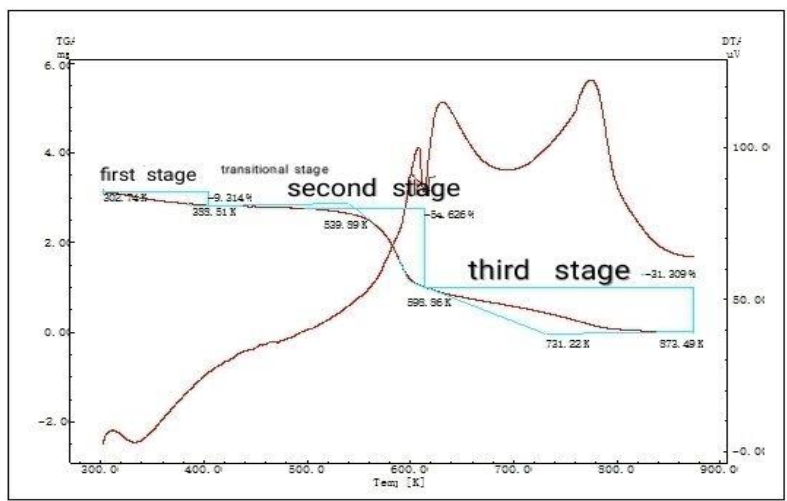

Figure 3. Analytical diagram of thermal analysis image

As the temperature increases, the first stage is the dehydration stage, and the temperature at which the reaction starts is $300 \mathrm{~K} \sim 388.51 \mathrm{~K}$, that is, $30^{\circ} \mathrm{C}-115.00^{\circ} \mathrm{C}$. The weight loss rate of the Tartary buckwheat flour in this stage is $9.314 \%$. 
The DTA curve in the transition stage is relatively stable, the mass change is not obvious, the reaction temperature is: $388.51 \mathrm{~K}-539.89 \mathrm{~K}$, namely $115.00^{\circ} \mathrm{C}-266.74^{\circ} \mathrm{C}$. At this stage, there is no significant mass change in the Tartary buckwheat flour, and the temperature range is a suitable for processing.

The second stage is the stage where the weight loss of Tartary buckwheat flour is the largest, and the reaction temperature is $539.89 \mathrm{~K}-593.86 \mathrm{~K}$, namely $266.74^{\circ} \mathrm{C}-320.74^{\circ} \mathrm{C}$. At this stage, the weight loss rate of Tartary buckwheat flour is the largest in the whole thermal decomposition process, reaching $54.626 \%$. The processing temperature should be lower than this temperature range, and this paper mainly studies the kinetic model of this stage.

In the third stage, the Tartary buckwheat flour undergoes carbonization reaction and other reactions, and the weight loss is $31.309 \%$. The reaction temperature is $593.86 \mathrm{~K} \sim 873.49 \mathrm{~K}$, namely $320.74^{\circ} \mathrm{C} \sim 600.34^{\circ} \mathrm{C}$. The third stage actually contains two reactions. Because the temperature is too high at this stage, such high temperature processing is generally not used in the actual processing, so we merge these two reactions into the third stage.

In summary, the free water content in the Tartary buckwheat flour can be roughly calculated to be $9.314 \%$, and the temperature at which the Tartary buckwheat flour begins to decompose largely is $539.87 \mathrm{~K}$, namely $266.74{ }^{\circ} \mathrm{C}$. The temperature should be controlled during the processing of Tartary buckwheat flour products so that the processing temperature of Tartary buckwheat flour is lower than this temperature.

\subsection{Derivation of thermokinetics parameter solving formula}

\subsubsection{Derivation of reaction kinetics formula}

According to the mass equation, the reaction rate formula can be expressed as:

$$
\frac{d \alpha}{d T}=K(T) \times f(\alpha) \times \frac{1}{\beta}
$$

Try out : $\alpha$ is conversion rate

$\mathrm{T}$ is temperate, the unit is $K$

$\beta$ is heating rate, the unit is ${ }^{\circ} \mathrm{C} / \mathrm{min}$

According to Arrhenius equation, there is [9]:

$$
\mathrm{K}(\mathrm{T})=\mathrm{A} \exp \left(-\frac{E}{R T}\right)
$$

where: A is the frequency factor; $\mathrm{E}$ is the activation energy, the unit is $\mathrm{J} / \mathrm{mol}$; $\mathrm{R}$ is the molar gas constant; $\mathrm{T}$ is the thermokinetic temperature.

Substitute (3) into (2) to get:

$\frac{d \alpha}{d T}=\frac{A}{\beta} \exp \left(-\frac{E}{R T}\right) \times f(\alpha)$

Separate variables and integrate, then we can get:

$\int_{0}^{\alpha} \frac{d \alpha}{f(\alpha)}=\int_{0}^{T} \frac{A}{\beta} \exp \left(-\frac{E}{R T}\right) d T$
Unfortunately, the integral on the right side of the equation does not converge in the right domain of $\mathrm{T}=0$, so an accurate analytical solution cannot be obtained, therefore the StavaSestak integral formula is used to approximate it.

\subsubsection{Kinetics mode function}

$G(\alpha)=\int_{0}^{\alpha} \frac{d \alpha}{f(\alpha)}$ Order:

$G(\alpha)$ is a kinetics mode function. The correct choice of the mode function has a very important influence on the solving of the kinetic parameters [10]. This paper compares the commonly used 30 [11] kinds of solid-phase non-isothermal kinetics mode functions, as shown in Table 1:

Table 1. Solid phase non-isothermal kinetic mode function table

\begin{tabular}{ll}
\hline Function No. & Kinetics mode function \\
\hline 1 & $\alpha^{2}$ \\
2 & $\alpha+(1-\alpha) \ln (1-\alpha)$ \\
3 & $(1-2 / 3 \alpha)-(1-\alpha)^{2 / 3}$ \\
4,5 & {$\left[1-(1-\alpha)^{1 / 3}\right]^{\mathrm{n}}(\mathrm{n}=2,1 / 2)$} \\
6 & {$\left[1-(1-\alpha)^{1 / 2}\right]^{1 / 2}$} \\
7 & {$\left[(1+\alpha)^{1 / 3}-1\right]^{2}$} \\
8 & {$\left[1 /(1+\alpha)^{1 / 3}-1\right]^{2}$} \\
9 & $-\ln (1-\alpha)$ \\
$10,11,12,13,14,15,16$ & {$[-\ln (1-\alpha)]^{\mathrm{n}}(\mathrm{n}=2 / 3,1 / 2,1 / 3,4,1 / 4,2,3)$} \\
$17,18,19,20,21,22$ & $1-(1-\alpha)^{\mathrm{n}}(\mathrm{n}=1 / 2,3,2,4,1 / 3,1 / 4)$ \\
$23,24,25,26,27$ & $\alpha^{\mathrm{n}}(\mathrm{n}=1 / 2,3,2,4,1 / 3,1 / 4)$ \\
28 & $(1-\alpha)^{-1}$ \\
29 & $(1-\alpha)^{-1}-1$ \\
30 & $(1-\alpha)^{-1 / 2}$ \\
\hline
\end{tabular}

Firstly, the 30 kinds of kinetic mode functions were respectively substituted into the Stava-Sestak equation to calculate the apparent activation energy and frequency factor respectively. Compared with the FWO method, the kinetic mode functions conforming to the Stava-Sestak method were selected, and then the pattern matching method was adopted to select a set of kinetic mode functions with the best linear fit, and the mechanism function calculated by this kinetic mode function was taken as the most probable mechanism function for the thermal decomposition of Tartary buckwheat flour to establish the thermal decomposition kinetics equation and to give theoretical predictions of the thermal decomposition reaction of Tartary buckwheat flour.

\subsubsection{Stava-Sestak method}

The Stava-Sestak method $[12,13]$ calculates the kinetic parameters by substituting various possible kinetic mode functions into the Stava-Sestak equation and calculating the regression equation, then it combines with the kinetic parameters obtained by the FWO method and the linear fit of the regression equation to select the best most approximate mechanism function.

By approximately solving formula (5) we can get formula of the Stava-Sestak method:

$\lg G(\alpha)=\lg \frac{A E}{R \beta}-2.315-\frac{0.4567 \mathrm{E}}{R T}$

By substituting the 30 kinds of kinetic mode functions of Table 1 into the $G(\alpha)$ of above formula, a linear regression 
equation of $\lg G(\alpha)$ and $1 / \mathrm{T}$ is obtained by using the least squares method:

$$
y=a x-b
$$

Then according to the linear regression equation there are:

$$
\begin{aligned}
& \mathrm{E}=\mathrm{a} \times \mathrm{R} \div 0.4567 \\
& \lg A=b+2.315+\lg \beta+\lg R-\lg E
\end{aligned}
$$

\subsubsection{FWO method}

By approximately solving formula (6) we can get the FWO equation [14]:

$$
\lg (\beta)=\lg \left(\frac{A E}{R G(\alpha)}\right)-2.315-0.456 \frac{E}{R T}
$$

where: $\beta$ is the heating rate; $A$ is the frequency factor; $R$ is the ideal gas constant, takes 8.314 here; $\mathrm{E}$ is the activation energy, the unit is $\mathrm{KJ} / \mathrm{mol}$; $\mathrm{T}$ is the reaction temperature, the unit is $\mathrm{K}$ (Kelvin).

By analyzing formula (9) we can find that, $\lg \beta$ has a linear relationship with $1 / \mathrm{T}$. In the actual calculation, $\alpha$ takes the temperatures of $0.10,0.20$, and 0.30 , respectively. The linear regression equation of the two are obtained by the least square method. The activation energy $\mathrm{E}$ is obtained from the slope, and the frequency factor $\mathrm{A}$ is obtained from the intercept.

An outstanding advantage of the FWO method over other methods is that it is not necessary to determine the kinetic mode function. For complex high molecular substances such as Tartary buckwheat flour, it is often impossible to determine its specific reaction, so it causes great difficulty for the determination of the kinetic mode function [15]. The apparent activation energy of Tartary buckwheat flour can be calculated more accurately using the FWO method. This outstanding advantage of FWO method is also often used to test the correctness of the hypothetical kinetic mode function. The appropriate kinetic mode function was screened by comparing with the results calculated by the Stava-Sestak method.

\subsection{Solving thermal analysis kinetic parameters}

\subsubsection{Selection of kinetic data}

Six temperature points are selected starting from $561.68 \mathrm{~K}$ with a gradient of $4 \mathrm{~K}$, and the conversion rate and temperature are read as shown in the following table.

Table 2. Kinetic data T- $\alpha$ table

\begin{tabular}{llllll}
\hline Conversion rate: $\alpha(\%)$ & 1.353 & 3.052 & 5.255 & 7.992 & 11.328 \\
\hline Temperature: $\mathrm{T}(\mathrm{K})$ & 559 & 563 & 567 & 571 & 575 \\
\hline
\end{tabular}

According to the Stava-Sestak equation, we can know that $\lg G(\alpha)$ and $1 / \mathrm{T}$ present a linear relationship, and the linear relationship equation can be obtained via pattern matching and the least squares method, then the activation energy $\mathrm{E}$ and the frequency factor A of the reaction can be solved by the slope

\begin{tabular}{|c|c|c|c|c|c|}
\hline Data points & Data point 1 & Data point 2 & Data point 3 & Data point 4 & Data point 5 \\
\hline Conversion rate $\alpha$ & 0.013530000 & 0.030520000 & 0.052550000 & 0.079920000 & 0.113280000 \\
\hline $1 / \mathrm{T}$ & 0.001788909 & 0.001776199 & 0.001763668 & 0.001751313 & 0.001739130 \\
\hline Function 1 & -3.737404407 & -3.030830941 & -2.558854559 & -2.194689050 & -1.891693519 \\
\hline Function 2 & -4.036466839 & -3.327397052 & -2.852140208 & -2.483827982 & -2.175666774 \\
\hline Function 3 & -4.689022505 & -3.979116468 & -3.502755731 & -3.133039911 & -2.823117122 \\
\hline Function 4 & -4.687708807 & -3.976130260 & -3.497561675 & -3.125038467 & -2.811591963 \\
\hline Function 5 & -1.171927202 & -0.994032565 & -0.874390419 & -0.781259617 & -0.702897991 \\
\hline Function 6 & -1.084127844 & -0.906546609 & -0.787317948 & -0.694712536 & -0.617009761 \\
\hline Function 7 & -4.687708807 & -3.976130260 & -3.497561675 & -3.125038467 & -2.811591963 \\
\hline Function 8 & -4.699434871 & -4.002511093 & -3.542838431 & -3.193643837 & -2.908445654 \\
\hline Function 9 & -1.865747503 & -1.50 & -1.267758158 & 2860 & -0.920 \\
\hline Function 10 & -1.243831668 & -1.005801522 & -0.845172106 & -0.719588573 & -0.613334360 \\
\hline Function 11 & -0.932873751 & -0.754351141 & -0.633879079 & -0.539691430 & -0.460000770 \\
\hline Function 12 & -0.62191 & -0.50290 & 86053 & 287 & -0.3 \\
\hline Function 13 & -7.462990010 & -6.034809130 & -5.071032633 & -4.317531440 & -3.680006158 \\
\hline Function 14 & & & & & 000385 \\
\hline on 15 & -3.731495005 & -3.017404565 & -2.535516317 & -2.1587 & -1.840003079 \\
\hline Function 16 & -5.597242508 & -4.526106847 & -3.803274475 & -3.238148580 & -2.760004618 \\
\hline Function 17 & -2.168255688 & -1.813093218 & -1.574635897 & -1.389425072 & -1.234019521 \\
\hline Function 18 & -1.397470203 & -1.051616295 & -0.825327988 & -0.655393881 & -0.518849042 \\
\hline Function 19 & -1.570620193 & -1.221063896 & -0.989960963 & -0.814025201 & -0.670139306 \\
\hline Function 20 & -1.275466020 & -0.933286462 & -0.711742216 & -0.547664786 & -0.418191629 \\
\hline Function 21 & -2.343854404 & -1.988065130 & -1.748780837 & -1.562519233 & -1.405795982 \\
\hline Function 22 & -2.468546799 & -2.112443830 & -1.872745316 & -1.685956806 & -1.528571870 \\
\hline Function 23 & -1.868702203 & -1.515415471 & -1.279427280 & -1.097344525 & -0.945846760 \\
\hline Function 24 & -2.803053305 & -2.273123206 & -1.919140919 & -1.646016787 & -1.418770139 \\
\hline Function 25 & -0.934351102 & -0.757707735 & -0.639713640 & -0.548672262 & -0.472923380 \\
\hline Function 26 & -0.622900734 & -0.505138490 & -0.426475760 & -0.365781508 & -0.315282253 \\
\hline Function 27 & -0.467175551 & -0.378853868 & -0.319856820 & -0.274336131 & -0.236461690 \\
\hline Function 28 & 0.005916118 & 0.013461146 & 0.023443700 & 0.036174410 & 0.052213496 \\
\hline Function 29 & -1.862786086 & -1.501954325 & -1.255983580 & -1.061170115 & -0.893633264 \\
\hline Function 30 & 0.002958059 & 0.006730573 & 0.011721850 & 0.018087205 & 0.026106748 \\
\hline
\end{tabular}
and intercept of the linear equation.

The results of the calculation are shown in Table 3 below:

Table 3. Solution results of 30 kinds of kinetics mode function

The linear regression equation for each kinetic mode function is obtained by least squares method, $\mathrm{y}=\mathrm{ax}+\mathrm{b}$.
The results are shown in Table 4: 
Table 4. Activation energy results obtained from the 30 kinds of kinetic mode functions

\begin{tabular}{|c|c|c|c|c|}
\hline Kinetic mode functions & $\mathrm{a}$ & $\mathrm{b}$ & $\mathrm{R}^{2}$ & $\mathrm{E}(\mathrm{kJ} / \mathrm{mol})$ \\
\hline Function 1 & -36432.00 & 61.578 & 0.9729 & 663.22 \\
\hline Function 2 & -36734.00 & 61.818 & 0.9737 & 668.72 \\
\hline Function 3 & -36836.00 & 61.348 & 0.9740 & 670.58 \\
\hline Function 4 & -37040.00 & 61.714 & 0.9745 & 674.29 \\
\hline Function 5 & -9260.10 & 15.428 & 0.9745 & 168.57 \\
\hline Function 6 & -9221.90 & 15.448 & 0.9741 & 167.88 \\
\hline Function 7 & -37040.00 & 61.714 & 0.9745 & 674.29 \\
\hline Function 8 & -35335.00 & 58.656 & 0.9700 & 643.25 \\
\hline Function 9 & -18674.00 & 31.609 & 0.9753 & 339.95 \\
\hline Function 10 & -12449.00 & 21.073 & 0.9753 & 226.62 \\
\hline Function 11 & -9336.90 & 15.805 & 0.9753 & 169.97 \\
\hline Function 12 & -6224.60 & 10.536 & 0.9753 & 113.31 \\
\hline Function 13 & -74695.00 & 126.440 & 0.9753 & 1359.78 \\
\hline Function 14 & -4668.50 & 7.902 & 0.9753 & 84.98 \\
\hline Function 15 & -37348.00 & 63.210 & 0.9753 & 679.90 \\
\hline Function 16 & -56022.00 & 94.828 & 0.9753 & 1019.85 \\
\hline Function 17 & -18444.00 & 30.896 & 0.9741 & 335.76 \\
\hline Function 18 & -17331.00 & 29.679 & 0.9678 & 315.50 \\
\hline Function 19 & -17768.00 & 30.288 & 0.9704 & 323.45 \\
\hline Function 20 & -16903.00 & 29.037 & 0.9651 & 307.71 \\
\hline Function 21 & -18520.00 & 30.857 & 0.9745 & 337.14 \\
\hline Function 22 & -18558.00 & 30.801 & 0.9747 & 337.83 \\
\hline Function 23 & -18216.00 & 30.789 & 0.9729 & 331.61 \\
\hline Function 24 & -27324.00 & 46.184 & 0.9729 & 497.42 \\
\hline Function 25 & -9108.10 & 15.395 & 0.9729 & 165.80 \\
\hline Function 26 & -6072.10 & 10.263 & 0.9729 & 110.53 \\
\hline Function 27 & -4454.00 & 7.697 & 0.9729 & 81.08 \\
\hline Function 28 & -925.42 & 1.659 & 0.9770 & 16.84 \\
\hline Function 29 & -19142.00 & 32.448 & 0.9775 & 348.47 \\
\hline Function 30 & -462.71 & 0.830 & 0.9770 & 8.42 \\
\hline
\end{tabular}

By comparison, it is found that the 30 kinds of kinetic mode functions have a significant effect on the solving of the activation energy of the Tartary buckwheat flour, correctly determining the kinetic mode function of Tartary buckwheat flour is especially important for the solving of kinetic parameters. As Tartary buckwheat flour is a kind of macromolecular organic matter, its reaction often cannot be directly determined, and its reaction order cannot be directly determined as well, so we can't determine its kinetic mode function by physical or chemical methods. Therefore, for the solving of kinetic mode functions of complex organic matter, the kinetic mode function is often replaced by using the approximate substitution method. This optimal substitution function is the most probable kinetic mode function, and its corresponding mechanism function is called the most probable mechanism function.

According to general experience, the activation energy is a positive number, and substances with an activation energy more than $400 \mathrm{KJ} / \mathrm{mol}$ are generally considered to be extremely resistant to chemical reactions at normal temperatures. Therefore, perform a preliminary screening on the obtained results and compare them with the results of the activation energy obtained by the FWO method below, and then solve the most probable kinetic mode function of the thermal decomposition of Tartary buckwheat flour.
3.4.2 Solving activation energy and frequency factor by FWO method

On the thermal analysis images of heating rates of $5^{\circ} \mathrm{C} / \mathrm{min}$, $10^{\circ} \mathrm{C} / \mathrm{min}, 15^{\circ} \mathrm{C} / \mathrm{min}$, and $20^{\circ} \mathrm{C} / \mathrm{min}$, respectively select temperature points of a conversion rate of $10 \%, 20 \%$, and $30 \%$, then the follow table is obtained:

Table 5. Data points calculated by FWO method

\begin{tabular}{|c|c|c|c|c|}
\hline \multicolumn{2}{|c|}{ Conversion rate $\beta\left({ }^{\circ} \mathrm{C} / \mathrm{min}\right)$} & $\mathrm{T}(\mathrm{K})$ & $1 / \mathrm{T}$ & $\operatorname{Lg} \beta$ \\
\hline \multirow{4}{*}{$10 \%$} & 5 & \multicolumn{3}{|c|}{568.200 .0017600 .698970004} \\
\hline & 10 & \multicolumn{3}{|c|}{573.860 .0017431 .000000000} \\
\hline & 15 & \multicolumn{3}{|c|}{579.350 .0017261 .176091259} \\
\hline & 20 & \multicolumn{3}{|c|}{583.450 .0017141 .301029996} \\
\hline \multirow{4}{*}{$20 \%$} & 5 & \multicolumn{3}{|c|}{575.510 .0017380 .698970004} \\
\hline & 10 & \multicolumn{3}{|c|}{583.000 .0017151 .000000000} \\
\hline & 15 & \multicolumn{3}{|c|}{589.940 .0016951 .176091259} \\
\hline & 20 & \multicolumn{3}{|c|}{594.550 .0016821 .301029996} \\
\hline \multirow{4}{*}{$30 \%$} & 5 & \multirow{2}{*}{\multicolumn{3}{|c|}{$\begin{array}{l}583.580 .0017140 .698970004 \\
589.690 .0016961 .000000000\end{array}$}} \\
\hline & 10 & & & \\
\hline & 15 & \multicolumn{3}{|c|}{597.200 .0016741 .176091259} \\
\hline & 20 & \multicolumn{3}{|c|}{602.120 .0016611 .301029996} \\
\hline
\end{tabular}

Substitute into the FWO equation and draw a diagram: 


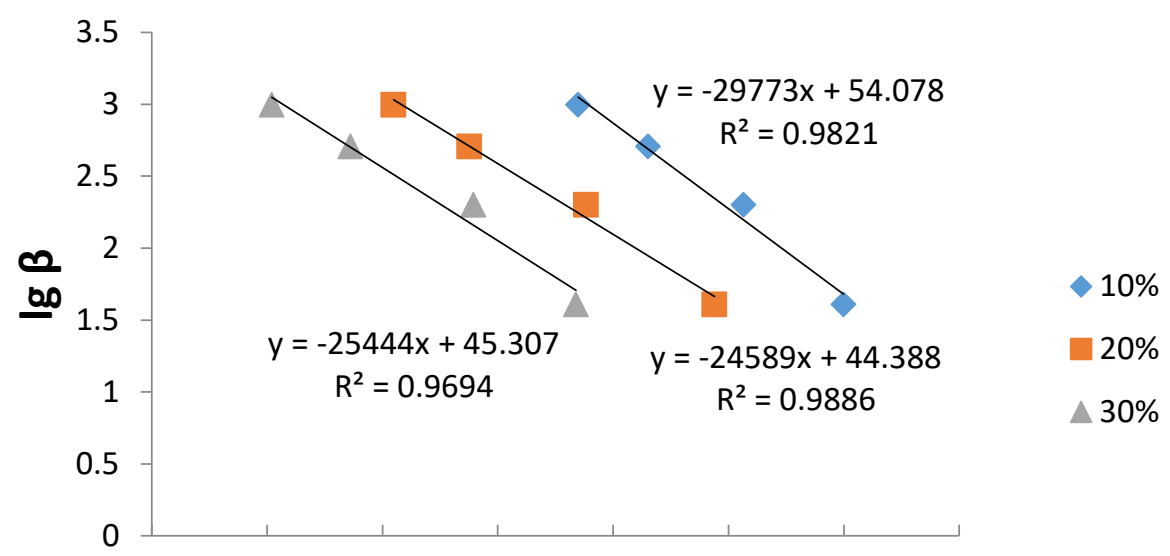

0.001640.001660.00168 0.0017 0.001720.001740.001760.00178

$1 / \mathrm{T}$

Figure 4. FWO method results

According to FWO equation, there is:

$\lg (\beta)=\lg \left(\frac{A E}{R G(\alpha)}\right)-2.315-0.456 \frac{E}{R T}$

The calculation results are shown in the following table.

Table 6. FWO method calculation results

\begin{tabular}{|c|c|c|c|}
\hline $\begin{array}{c}\text { Conversion rate } \\
(\%)\end{array}$ & $\begin{array}{c}\text { Activation energy } \\
\mathrm{E}(\mathrm{KJ} / \mathrm{mol})\end{array}$ & LgA & LnA \\
\hline $10 \%$ & 235.38 & 19.14 & 44.07 \\
\hline $20 \%$ & 194.40 & 19.52 & 44.95 \\
\hline $30 \%$ & 201.15 & 19.68 & 45.32 \\
\hline
\end{tabular}

Comparison of the activation energies of different conversion rates shows that, the activation energies of the three-stage conversion rate are not the same. The activation energy of the first stage is the largest, which is because the complexity of the components of Tartary buckwheat flour and its reactions has led to this reaction not being an elementary reaction, but a result caused by many simultaneous reactions. Since the most concerned issue in food processing should be the temperature at which the Tartary buckwheat flour begins to decompose, so here we select the activation energy of the first stage reaction with a conversion rate of $10 \%$ for our research, that is: $\mathrm{E}=235.38 \mathrm{KJ} / \mathrm{mol}, \mathrm{LnA}=44.07$.

\subsection{Determination of the most probable mechanism function}

Compare the 30 kinds of kinetic mode functions and solve their activation energies and errors as shown in Table 7, the allowable range of error is:

$\left|\frac{E_{0}-E_{s}}{E_{0}}\right| \leq 0.3$

The activation energies and frequency factors obtained by the 30 kinetic mode functions are compared with the results obtained by the FWO method. It can be seen that the influence of the selection of the kinetic mode function on the activation energy solution is quite significant, and determining an appropriate kinetic mode function is very important for solving the kinetic parameters of the Tartary buckwheat flour.

Table 7. Error of activation energy solved by 30 kinds of kinetic mode functions

\begin{tabular}{cccc}
\hline Kinetic mode functions & $\mathrm{R}^{2}$ & $\mathrm{E}(\mathrm{kJ} / \mathrm{mol})$ & $\mathrm{E}$ Error \\
\hline Function 1 & 0.9729 & 663.22 & 1.817634 \\
Function 2 & 0.9737 & 668.72 & 1.840990 \\
Function 3 & 0.9740 & 670.58 & 1.848879 \\
Function 4 & 0.9745 & 674.29 & 1.864656 \\
Function 5 & 0.9745 & 168.57 & 0.283828 \\
Function 6 & 0.9741 & 167.88 & 0.286783 \\
Function 7 & 0.9745 & 674.29 & 1.864656 \\
Function 8 & 0.9700 & 643.25 & 1.732792 \\
Function 9 & 0.9753 & 339.95 & 0.444238 \\
Function 10 & 0.9753 & 226.62 & 0.037200 \\
Function 11 & 0.9753 & 169.97 & 0.277889 \\
Function 12 & 0.9753 & 113.31 & 0.518592 \\
Function 13 & 0.9753 & 1359.78 & 4.776876 \\
Function 14 & 0.9753 & 84.98 & 0.638940 \\
Function 15 & 0.9753 & 679.90 & 1.888477 \\
Function 16 & 0.9753 & 1019.85 & 3.332715 \\
Function 17 & 0.9741 & 335.76 & 0.426450 \\
Function 18 & 0.9678 & 315.50 & 0.340371 \\
Function 19 & 0.9704 & 323.45 & 0.374169 \\
Function 20 & 0.9651 & 307.71 & 0.307270 \\
Function 21 & 0.9745 & 337.14 & 0.432328 \\
Function 22 & 0.9747 & 337.83 & 0.435267 \\
Function 23 & 0.9729 & 331.61 & 0.408817 \\
Function 24 & 0.9729 & 497.42 & 1.113225 \\
Function 25 & 0.9729 & 165.80 & 0.295584 \\
Function 26 & 0.9729 & 110.53 & 0.530387 \\
Function 27 & 0.9729 & 81.08 & 0.655530 \\
Function 28 & 0.9770 & 16.84 & 0.928428 \\
Function 29 & 0.9775 & 348.47 & 0.480433 \\
Function 30 & 0.9770 & 8.42 & 0.964214 \\
\hline & & & \\
\hline
\end{tabular}

By comparison we find that the kinetic mode functions that satisfy the conditions are: No. 4, 6, 10,11. Then by comparing the linear fits, we find that the No.10 function has the highest linear fit and its activation energy is also significantly closer to that obtained by the FWO method. Therefore, function 10 
is selected as the kinetic mode function for the Tartary buckwheat flour, that is:

$$
\mathrm{G}(\alpha)=[-\ln (1-\alpha)]^{2 / 3}
$$

The most probable mechanism function of the thermal decomposition of Tartary buckwheat flour calculated by above formula is:

$$
f(\alpha)=\frac{3}{2}[-\ln (1-\alpha)]^{1 / 3} \times(1-\alpha)
$$

\subsection{Establishment of the kinetic equation of Tartary buckwheat flour}

The activation energy calculated by the FWO method is: $\mathrm{E}=235.38 \mathrm{KJ} / \mathrm{mol}$

The frequency factor is: $\ln A=44.07$.

Using Stava-Sestak method, the activation energies calculated by 30 kinds of kinetic mode functions are compared and then perform logical analysis of its linear fitness to screen out the most probable mechanism function [16].

In summary, the kinetic equation is obtained as follows: $\frac{d \alpha}{d t}=\exp \left\{44.07-\frac{235384.32}{R T}\right\} \times \frac{3}{2}(1-\alpha) \cdot[-\ln (1-\alpha)]^{1 / 3}$

Separate the variable and integrate both sides of the equation simultaneously to get:

$[-\ln (1-\alpha)]^{\frac{2}{3}}=\exp \left\{44.07-\frac{235384.32}{R T}\right\} \times t$

A thermal analysis kinetic model for the Tartary buckwheat flour is obtained, then predict the kinetic model of the Tartary buckwheat flour.

\subsection{Verification of the thermal decomposition kinetic model of the Tartary buckwheat flour}

Take the $560 \mathrm{~K}-570 \mathrm{~K}$ temperature range from the TGA diagram and read the derivative of conversion rate with respect to time, and then compare it with the theoretical value calculated by the kinetic model, as shown in the following table:

\begin{tabular}{|c|c|c|c|c|}
\hline time (sec) & $\mathrm{T}(\mathrm{K})$ & Actual results & Model results & Accuracy \\
\hline 1535 & 560.935706 & 0.001051424 & 0.000901308 & 0.857226 \\
\hline 1536 & 561.081396 & 0.001052325 & 0.000917051 & 0.871452 \\
\hline 1537 & 561.280493 & 0.001057285 & 0.000938054 & 0.887229 \\
\hline 1538 & 561.439795 & 0.001071713 & 0.000956029 & 0.892057 \\
\hline 1539 & 561.599493 & 0.001075771 & 0.000974079 & 0.905471 \\
\hline 1540 & 561.795813 & 0.001103724 & 0.000995663 & 0.902094 \\
\hline 1541 & 561.943396 & 0.00112717 & 0.001013561 & 0.899209 \\
\hline 1542 & 562.12171 & 0.001160083 & 0.001034733 & 0.891947 \\
\hline 1543 & 562.319312 & 0.001193898 & 0.001057676 & 0.885902 \\
\hline 1544 & 562.4578 & 0.001202915 & 0.001075896 & 0.894407 \\
\hline 1545 & 562.653601 & 0.001203817 & 0.001100156 & 0.91389 \\
\hline 1546 & 562.842291 & 0.001199308 & 0.001123523 & 0.936809 \\
\hline 1547 & 562.978888 & 0.001212384 & 0.00114199 & 0.941938 \\
\hline 1548 & 563.179694 & 0.001217343 & 0.001167899 & 0.959383 \\
\hline 1549 & 563.34809 & 0.001225459 & 0.001190811 & 0.971727 \\
\hline 1550 & 563.526709 & 0.001235378 & 0.001214422 & 0.983037 \\
\hline 1551 & 563.695105 & 0.001249806 & 0.00123803 & 0.990578 \\
\hline 1552 & 563.858893 & 0.001282268 & 0.001261672 & 0.983938 \\
\hline 1553 & 564.020514 & 0.001306615 & 0.001285007 & 0.983463 \\
\hline 1554 & 564.221594 & 0.00133547 & 0.001313467 & 0.983524 \\
\hline 1555 & 564.369208 & 0.001354407 & 0.001336646 & 0.986887 \\
\hline 1556 & 564.539496 & 0.001377852 & 0.00136268 & 0.988989 \\
\hline 1557 & 564.735297 & 0.001377852 & 0.001391827 & 0.989857 \\
\hline 1558 & 564.890692 & 0.001377852 & 0.001417177 & 0.971459 \\
\hline 1559 & 565.057104 & 0.001373794 & 0.001443886 & 0.948979 \\
\hline 1560 & 565.250494 & 0.001368384 & 0.001474303 & 0.922596 \\
\hline 1561 & 565.405096 & 0.00138191 & 0.001500079 & 0.914488 \\
\hline 1562 & 565.584509 & 0.001387771 & 0.001530108 & 0.897435 \\
\hline 1563 & 565.761115 & 0.001411216 & 0.001559852 & 0.894675 \\
\hline 1564 & 565.920599 & 0.001425193 & 0.001587466 & 0.88614 \\
\hline 1565 & 566.110999 & 0.001449089 & 0.001620617 & 0.88163 \\
\hline 1566 & 566.273901 & 0.001467124 & 0.001650111 & 0.875275 \\
\hline 1567 & 566.437506 & 0.001495979 & 0.001679962 & 0.877015 \\
\hline 1568 & 566.625098 & 0.001519875 & 0.001714005 & 0.872273 \\
\hline 1569 & 566.788794 & 0.001528442 & 0.001745705 & 0.857853 \\
\hline
\end{tabular}

Table 8. Comparison between model calculation results and actual values

It can be seen from the data in above table that the solving of kinetic equations has a good simulation of the decomposition of Tartary buckwheat flour. In the temperature range of $560 \mathrm{~K} \sim 570 \mathrm{~K}$, the reaction rate obtained by TGA images and the values of the model are on the same order of magnitude, the accurate ranges are all above $85 \%$, so the 
established kinetic mode function is relative reliable. Also, we can see from the table that the accuracy decreases with the increase of temperature, this may be determined by the complex components of the Tartary buckwheat flour itself. This conclusion has also been verified when using FWO method, under a higher temperature, the activation energy, frequency factor and most probable mechanism function have changed [17-21]. However, in food processing, what we concern most is the first stage reaction, so we will not further solve and discuss the reactions in later stages here.

\section{DISCUSSION}

\subsection{Determination of proper processing temperature}

By thermal analysis, we can get the temperature at which the Tartary buckwheat flour begins to decompose is $539.89 \mathrm{~K}$ or $266.74{ }^{\circ} \mathrm{C}$. In order to reduce the reaction of Tartary buckwheat flour during the actual production and processing, the processing temperature should be lower than this temperature.

\subsection{Determination of the best experimental conditions for the kinetic test of Tartary buckwheat flour}

By comparing the thermal analysis images obtained by different masses and different heating rate conditions, the best conditions for obtaining the thermal analysis test of the Tartary buckwheat flour by selecting sharp and obvious peak images are: the sample mass is $3.000 \mathrm{~g}$, and the heating rate is: $10{ }^{\circ} \mathrm{C} / \mathrm{min}$

\subsection{Determination of the thermal decomposition stages of Tartary buckwheat flour}

Through the thermal decomposition image of Tartary buckwheat flour, we can get that the decomposition of Tartary buckwheat flour can be divided into four stages: the initial dehydration stage, the transition stage, the first reaction stage, and the second reaction stage. Among them, the Tartary buckwheat flour in the transition stage is the most stable, and it is the optimal temperature range for processing, which is $115.00{ }^{\circ} \mathrm{C} \sim 266.74{ }^{\circ} \mathrm{C}$. In the first stage, the weight loss of the Tartary buckwheat flour is the most serious, and the onset temperature of the reaction is $266.74{ }^{\circ} \mathrm{C}$, and this temperature should be avoided in order to prevent mass loss during processing. The second stage is the final reaction stage of the Tartary buckwheat flour, in which the Tartary buckwheat flour begins to undergo carbonization and other reactions.

\subsection{Establishment of kinetic model for the thermal decomposition of Tartary buckwheat flour}

This paper uses the FWO method to calculate the activation energy of the thermal decomposition reaction of Tartary buckwheat flour to be: $235.38 \mathrm{KJ} / \mathrm{mol}$, frequency factor: $\mathrm{LnA}$ $=44.07$. By comparing the activation energies solved by the 30 kinds of kinetic mode functions and the activation energy solved by the FWO method, and by comparing the linear fits of the 30 kinetic mode functions, we can find that the No.10 kinetic mode function is the most probable kinetic mode function for the thermal decomposition of Tartary buckwheat flour, that is:
$\mathrm{G}(\alpha)=[-\ln (1-\alpha)]^{2 / 3}$

By calculation we can get the most probable mechanism function of the thermal decomposition of Tartary buckwheat flour as:

$f(\alpha)=\frac{3}{2}[-\ln (1-\alpha)]^{1 / 3} \times(1-\alpha)$

Further, the thermal decomposition kinetic model of Tartary buckwheat flour is obtained as follows:

$$
\begin{aligned}
& \frac{d \alpha}{d t}=\exp \left\{44.07-\frac{235384.32}{R T}\right\} \times \\
& \frac{3}{2}(1-\alpha) \bullet[-\ln (1-\alpha)]^{1 / 3}
\end{aligned}
$$

Separate the variable from the above formula and integrate to get:

$$
[-\ln (1-\alpha)]^{2 / 3}=\exp \left\{44.07-\frac{235384.32}{R T}\right\} t
$$

It is found through verification that this model has a good simulation effect in the temperature range of $560 \sim 570 \mathrm{~K}$, which has certain guiding significance for the actual production.

\section{REFERENCES}

[1] Wang JB. (2013). Development and quality analysis of Tartary buckwheat compound yogurt. Xihua University, 2013

[2] Yin LG, Zhong G, Zeng FK, Min YP. (2002). Buckwheat processing. Cereals \& Oils (9): 39-41. http://doi.org/ 10.3969/j.issn.1008-9578.2002.09.018

[3] Pu YL. (2012). Overview of application of thermal analysis techniques. Guangdong Chemical Industry 39(06): 45, 44. http://doi.org/10.3969/j.issn.10071865.2012.06.022

[4] Zhu JP. (2015). Study on thermal analysis for the distinguish of black buckwheat tea and raw buckwheat. Food Research and Development 36(09): 13-16. http://doi.org/ 10.3969/j.issn.1005-6521.2015.09.004

[5] Ren YL, Cheng BW, Xu L, Li ZH, Jiang AB, Lu YC. (2009). Non-isothermal decomposition kinetics of novel fire retarded polyacrylonitrile copolymer in air. Acta Chimica Sinica 67(18): 2127-2132. http://doi.org/ 10.3321/j.issn:0567-7351.2009.18.012

[6] Kissinger HE. (1956). Variation of peak temperature with heating rate in differential thermal analysis. Journal of Research of the National Bureau of Standards 57(4): 217-221.

[7] Moon C, Sung Y, Ahn S, Kim T, Choi G, Kim D. (2013). Effect of blending ratio on combustion performance in blends of biomass and coals of different ranks. Experimental Thermal and Fluid Science (47): 232-240. http://doi.org/10.1016/j.expthermflusci.2013.01.019

[8] Zhang K, Zhang K, Cao Y, Pan W. (2013). Cocombustion characteristics and blend-ing optimization of 
tobacco stem and high-sulfur bituminous coal based o-n thermogravimetric and mass spectrometry analyses. Bioresources Technology 131: 325-332. http://doi.org/10.1016/j.biortech.2012.12.163

[9] Jiang C. (2015). Optimization of coal spontaneous combustion mechanism function by artificial fish swarm algorithm. Liaoning University of Engineering and Technology.

[10] Zou SP, Wu YL, Yang MD, Li C, Tong JM. (2009) Pyrolysis characteristics and kinetics of the marine microalgae Dunaliella tertiolecta using thermogravimetric analyzer. Bioresource Technology 101(1) 359-365. http://doi.org/10.1016/j.biortech.2009.08.020

[11] Wang WW. (2012). biomass characteristics research based on TGA test and BP neural network. North China Electric Power University.

[12] Lai YH, Lu MX, Ma CY, Shi MH. (2001). Research on pyrolysis characteristics of agricultural residues under liner heating temperature. Journal of Combustion Science and Technology 7(3): 245-248. http://doi.org/10.3321/j.issn:1006-8740.2001.03.009

[13] Liu RH, Yuan HR, Xu L. (2007). Kinetic study of maize straw pyrolysis. Acta Energiae Solaris Sinica 28(5): $527-$ 531. 0096.2007.05.014

[14] Liu ZH, Xu GH, Zhang HL. (2006). Thermal analysis instrument. Beijing: Chemical Industry Press, 2006

[15] Hu RZ, Shi QZ. (2001). Thermal analysis dynamics. Beijing: Science Press.

[16] Wang MF, Jiang EC, Zhou L. (2009). Kinetic analysis of cornstalk pyrolysis. Transactions of The Chinese Society of Agricultural Engineering 25(2): 204-207.

[17] Alonso M, Borrego A G, Alvarez D, Menéndez R. (2001). Reactivity study of chars obtained at different temperatures in relation to their petrographic characteristics. Fuel Processing Technology 69(3): 257272. http://doi.org/10.1016/S0378-3820(00)00146-6

[18] Zhao M, Wu W, Lu M, Wei XY. (2002). Pyrolysis kinetics of rice straw. Transactions of The Chinese
Society of Agricultural Engineering 18(1): 107-110. http://doi.org/ 10.1007/s11769-002-0038-4

[19] Stenseng M, Jensen A, Dam-Johansen K. (2001). Investigation of biomass pyrolysis by thermogravimetric analysis and differential scanning calorimetry. Journal of Analytical and Applied Pyrolysis 58(1): 765-780. http://doi.org/ 10.1016/S0165-2370(00)00200-X

[20] Jiang L, Wang YZ, Yu T, Wu T. (2013). Mechanism of synergistic corrosion of sulfur and chlorine components in biomass mixed coal combustion. Power Station System Engineering (03): 1-4

[21] Liu HX, Cao YB, Li YX. (2009). Application of thermal analysis in the analysis of edible spices and flavors. Food Science 30(17): 349-354. http://doi.org/10.1007/978-3540-85168-4_52

\section{APPENDIX}

\section{Symbol description}

E activation energy, $\mathrm{KJ} \bullet \mathrm{mol}^{-1}$

$\mathrm{G}(\alpha)$ kinetics mode function

TGA curve: thermogravimetric analysis curve

DTA curve: differential thermal analysis curve

$\mathrm{K}$ thermokinetic temperature, unit in $\mathrm{K}$ (Kelvin)

$\alpha \quad$ solid sample mass conversion rate

$\mathrm{T}$ temperature, $\mathrm{K}$

$\beta \quad$ differential thermal analysis heating rate: ${ }^{\circ} \mathrm{C} / \mathrm{min}$

$\mathrm{f}(\alpha) \quad$ reaction mechanism function

$\mathrm{R} \quad$ ideal gas constant, $8.314{\mathrm{KJ} \bullet \mathrm{mol}^{-1}}^{-1}$

Exp natural logarithm

$\mathrm{E}_{0}$ activation energy calculated by the FWO method, $\mathrm{KJ} \bullet \mathrm{mol}^{-1}$

$E_{s} \quad$ activation energy calculated by the Stava-Sestak method, $\mathrm{KJ} \mathrm{mol}^{-1}$

$\mathrm{t}$ time, unit: second 